\title{
Mixed-Integer Linear Programming Framework for Max-Min Power Control with Single-Stage Interference Cancellation
}

\author{
Eleftherios Karipidis, Di Yuan and Erik G. Larsson
}

\section{Linköping University Pre Print}

N.B.: When citing this work, cite the original article.

(C2011 IEEE. Personal use of this material is permitted. However, permission to reprint/republish this material for advertising or promotional purposes or for creating new collective works for resale or redistribution to servers or lists, or to reuse any copyrighted component of this work in other works must be obtained from the IEEE.

Eleftherios Karipidis, Di Yuan and Erik G. Larsson, Mixed-Integer Linear Programming Framework for Max-Min Power Control with Single-Stage Interference Cancellation, 2011, Proceedings of the IEEE International Conference on Acoustics, Speech and Signal Processing (ICASSP) . 


\title{
MIXED-INTEGER LINEAR PROGRAMMING FRAMEWORK FOR MAX-MIN POWER CONTROL WITH SINGLE-STAGE INTERFERENCE CANCELLATION
}

\author{
Eleftherios Karipidis ${ }^{\star}$, Di Yuan ${ }^{\dagger}$, and Erik G. Larsson ${ }^{\star}$ \\ karipidis@isy.liu.se, diyua@itn.liu.se, erik.larsson@isy.liu.se \\ * Dept. of Electrical Engineering (ISY), Linköping University, SE-581 83 Linköping, Sweden \\ ${ }^{\dagger}$ Dept. of Science \& Technology (ITN), Linköping University, SE-601 74 Norrköping, Sweden
}

\begin{abstract}
We consider a wireless network comprising a number of mutuallyinterfering links. We study the transmit power control problem that determines the egalitarian signal-to-interference-plus-noise ratio under a novel setup. Namely, we assume that the receivers have multiuser detection capability, which enables decoding and cancellation of the interference, when it is strong enough. Determining the interference terms that can be cancelled is a combinatorial problem, which is intertwined with the power control problem. We propose a mixed-integer linear programming framework that jointly solves these problems optimally, using off-the-shelf algorithms. We illustrate with a simulation result the merit of the novel approach against the conventional one that precludes interference cancellation.
\end{abstract}

Index Terms - Combinatorial optimization, interference cancellation, linear programming, power control, multiuser detection

\section{INTRODUCTION}

The fundamental aspects of power control for wireless networks can be understood by studying a generic model comprising $K$ links (single-antenna transmitter-receiver pairs). The transmissions take place concurrently over the same frequency channel and the links operate in the vicinity of one other. Hence, due to the broadcast nature of the wireless medium, each receiver listens to a superposition of the desired signal and all other $K-1$ transmitted signals, which constitute interference. The setup under consideration is called a multiterminal interference channel. The capacity region of the interference channel is only known in a few special cases, e.g., see $[1,2]$ for recent contributions. Two basic facts are the following. When the interference is very weak, it can simply be treated as additive noise. When the interference is strong enough, it may be decoded and subtracted off from the received signal, leaving an interferencefree signal containing only the signal of interest plus thermal noise.

In previous studies of power control, the interfering signals are accounted as additive noise at the receivers, i.e., no attempt is made to decode them. Under this assumption, the rate that a link can support is monotonously increasing with the signal-to-interference-andnoise ratio (SINR) that the receiver experiences. Thus, it is equivalent to optimize the SINR's in lieu of the rates. This is preferable

This work has been performed in the framework of the European research project SAPHYRE, which is partly funded by the European Union under its FP7 ICT Objective 1.1 - The Network of the Future. This work has been supported in part by the Swedish Research Council (VR), the Swedish Foundation of Strategic Research (SSF), and the Excellence Center at Linköping-Lund in Information Technology (ELLIIT). E. G. Larsson is a Royal Swedish Academy of Sciences (KVA) Research Fellow supported by a grant from Knut and Alice Wallenberg Foundation. because it leads to linear programming (LP) optimization problems, which are solved very efficiently.

In this work, we make the paradigm-changing assumption that the receivers have multiuser detection capability. That is, they have sufficient information (codebooks and modulation levels) to potentially decode any interfering signal [3]. The prerequisite for successful decoding is that the signal on the respective crosstalk link is received sufficiently strong. The decoded signal is subsequently subtracted from the total interference sum. The effect is that the achievable rates now depend on the sum of the residual, i.e., undecoded, interference. Hence, to increase the achievable rates in interferencelimited environments, it might be favorable to transmit at high power levels, in order to enable interference cancellation (IC). This is in contrast to the conventional approaches that, in an effort to decrease the overall interference, minimizes the transmit powers.

Apparently, a novel power control problem emerges at the transmitters when IC is possible at the receivers. This problem is intertwined with the selection of the actual crosstalk links and the order thereof to perform successive IC (SIC). Determining the optimal SIC order jointly with the enabling transmit powers is a hard combinatorial problem. Henceforth, we restrict our interest to the special case where each receiver can decode at most one interfering signal and denote it single-stage IC. This gives rise to a relatively simple combinatorial problem at the receivers, which is coupled with a power control problem at the transmitters. We propose a mixed-integer linear programming (MILP) framework that tackles the joint problem of power control with single-stage IC. This formulation enables us to find the globally-optimum solution of the problem efficiently, for the vast majority of instances, using off-the-shelf algorithms.

Herein, we focus on the max-min formulation of the power control problem whose objective is to maximize, under a limited power budget, the SINR that all links can simultaneously achieve. Without IC, max-min power control is a quasi-LP problem. With IC, we prove herein that it becomes an NP-hard problem. Due to space limitations, we do not consider the alternative formulation which minimizes the aggregate power expenditure while ensuring some predetermined SINR thresholds for all links. An important difference between the two aforementioned formulations is that the max-min formulation is always feasible, whereas the alternative one may be infeasible for some instances. Infeasibility gives rise to admission control, which is an NP-hard combinatorial problem on its own right.

\section{PRELIMINARIES}

Under the common assumption that the receivers treat the interference as additive noise, the maximum achievable link rate is dictated by the SINR experienced at the receiver. That is, the communication 
quality on the $k$ th link is quantified by the term ${ }^{1}$

$$
\mathrm{SINR}_{k} \triangleq \frac{G_{k k} p_{k}}{\sum_{\ell \neq k} G_{\ell k} p_{\ell}+1} .
$$

In (1), $p_{k}$ is the transmit power on the $k$ th link and $G_{\ell k}$ is the gain of the channel between the $\ell$ th transmitter and the $k$ th receiver. The $k$ th transmit power can be adjusted up to a bound $P_{k}$, which is typically determined by regulatory and hardware constraints. The channel gains include the effects of propagation loss, shadowing and fading. Here, for notation simplicity, the channel gains are also normalized with the noise variance. The noise is assumed to be AWGN with equal variance for all receivers.

From (1), it is seen that the SINR of the $k$ th link depends on all $K$ transmit powers. Boosting $p_{k}$ increases $\operatorname{SINR}_{k}$, but reduces $\operatorname{SINR}_{\ell} \forall \ell \neq k$. Hence, the power terms have to be jointly set and SINR optimization is a problem with conflicting objectives. A popular approach is to look for the egalitarian solution. This is achieved with the following max-min formulation

$$
\max _{\left\{p_{k} \in\left[0, P_{k}\right]\right\}_{k \in \mathcal{K}}} \min _{k \in \mathcal{K}} \operatorname{SINR}_{k}
$$

where $\mathcal{K} \triangleq\{1, \ldots, K\}$ is the set of all direct links. The objective function (2) maximizes (over the feasible set of transmit powers) the minimum (over the $K$ links) received SINR. Problem (2) has attracted considerable interest in the recent past; see e.g. [4] and references therein.

A possible way to solve problem (2) is by means of standard convex optimization techniques. Specifically, introducing an auxiliary positive-real scalar variable, say $t,(2)$ can be equivalently reformulated as

$$
\begin{array}{lc} 
& \max _{t \in \mathbb{R}_{+},\left\{p_{k} \in\left[0, P_{k}\right]\right\}_{k \in \mathcal{K}}} t \\
\text { s.t. } & \operatorname{SINR}_{k} \geq t \quad \forall k \in \mathcal{K} .
\end{array}
$$

As evidenced from (4), the operational meaning of $t$ is that it bounds from below the SINR achieved by all links. The objective function (3) maximizes this threshold. For the optimum solution, all $K$ inequalities in (4) are tight, i.e. they are satisfied as equalities. Otherwise, it would be possible to further increase the objective value $t^{\star}$ of (3)-(4), by slightly decreasing the transmit power on the link that experiences SINR higher than $t^{\star}$, so that the SINR's on all other links increase. Hence, at optimum all $K$ SINR's are equalized to $t^{\star}$. For this reason, the max-min power control formulation (2) is often called SINR balancing, since it ensures the same SINR to all links.

Observing that the denominator of $\mathrm{SINR}_{k}$ is positive, we may multiply both sides of (4) with it and equivalently rewrite (3)-(4) as

$$
\begin{aligned}
& \max _{t \in \mathbb{R}_{+},\left\{p_{k} \in\left[0, P_{k}\right]\right\}_{k \in \mathcal{K}}} t \\
& \text { s.t. } \quad \sum_{\ell \neq k} G_{\ell k} p_{\ell} t-G_{k k} p_{k}+t \leq 0 \quad \forall k \in \mathcal{K},
\end{aligned}
$$

Problem (5)-(6) is quasi-LP, since the first term of (6) is bilinear (the variable $t$ is multiplied with the variables of interfering transmit powers). Problem (5)-(6) can be efficiently solved with a line search algorithm (e.g., bisection) on $t$. The search interval may be

\footnotetext{
${ }^{1}$ Throughout, we follow the convention that lowercase and uppercase letters denote real scalar variables and parameters, respectively. The first index determines the transmitter, whereas the second (if any) the receiver of a link.
}

initialized by the following conservative (i.e. loose) lower and upper bounds, respectively, on the common achievable SINR

$$
L:=\min _{k \in \mathcal{K}} \frac{G_{k k} P_{k}}{\sum_{\ell \neq k} G_{\ell k} P_{\ell}+1} \quad \text { and } \quad U:=\min _{k \in \mathcal{K}} G_{k k} P_{k} .
$$

In every iteration of the bisection algorithm, $t$ takes the value $(L+U) / 2$. Then, the formulation (5)-(6) denotes a feasibility problem, with respect to the transmit powers, consisting of the (now linear) inequalities (6) and the power bounds. Hence, this feasibility problem is a simple LP problem, which is solved very efficiently, even with matrix inversion since the inequalities are tight at optimum. Feasibility problems are optimization problems with constraints but without an objective function. Their solution is binary and answers the question whether the feasible set is empty or not. In the bisection algorithm, if a solution to the feasibility problem exists, then the lower bound is updated with the feasible value of $t$, i.e., $L:=t$. Otherwise, the upper bound is updated with the infeasible value of $t$, i.e., $U:=t$. The algorithm terminates when $U-L<\epsilon$ for a predetermined accuracy $\epsilon$. Since the search interval is halved after each iteration, the bisection algorithm converges after $\left\lceil\log _{2} \frac{U-L}{\epsilon}\right\rceil$ number of steps, where $L$ and $U$ here are the initial bounds. Typically, a small number of iterations suffices to find a solution which is accurate enough for engineering purposes.

\section{MAX-MIN POWER CONTROL WITH SINGLE-STAGE INTERFERENCE CANCELLATION}

In this section, we revisit the max-min power control problem under a novel setting. Specifically, we now assume that the receivers can potentially decode any interfering signal provided that it is received strong enough. We further assume that each receiver selects at most one crosstalk link to cancel the interference from. We formulate the joint optimization problem of selecting the transmission powers and the interference terms that the receivers cancel.

Towards this direction, we introduce the auxiliary binary variables $\left\{x_{m k} \in\{0,1\}\right\}_{m, k \in \mathcal{K}}$. The binary variable $x_{m k}$ models the capability of the $k$ th receiver to decode the signal stemming from the $m$ th transmitter. The value 1 means that it is possible to successfully decode, whereas 0 stands for the opposite event. When $x_{k k}=1$ the $k$ th receiver decodes only the desired signal (i.e., no IC is possible), whereas when $x_{m k}=1$ for any $m \neq k$, it first decodes the $m$ th interfering signal, subtracts it from the received signal and then decodes the desired one. The aforementioned scenarios are mutually exclusive; i.e., at any time only one of the $K$ variables that correspond to the $k$ th receiver can be equal to 1 .

Our goal is to formulate the novel power control problem in a way that preserves the main feature (the quasi-linearity) of conventional problem (3)-(4). To accomplish this we note that the binary variables $x_{m k}$ essentially express "if statements" in the sense that if $x_{m k}=1$ then certain set of SINR inequalities should be activated, and if $x_{m k}=0$ then the corresponding inequalities should be inactivated. These "if statements" are all of the form that an inequality $a>b$ should be enabled if and only if $x=1$. The trick is then to write this condition as $a+c(1-x)>b$, where $c$ is the largest value that $b-a$ can ever assume, because if $x=1$, the inequality reduces to $a>b$ and if $x=0$, it is always satisfied regardless of $a$ and $b$. Our way of formulating the problem makes extensive use of this trick, which we have successfully used before for other combinatorial power control problems $[5,6]$.

Using the auxiliary variables $\left\{x_{m k}\right\}_{m, k \in \mathcal{K}}$, we formulate the joint problem as 
subject to $\max _{\text {max }} \underset{\mathbb{R}_{+},\left\{p_{k} \in\left[0, P_{k}\right], x_{m k} \in\{0,1\}\right\}_{m, k \in \mathcal{K}}}{t}$

$$
\begin{array}{ll}
\frac{G_{k k} p_{k}+M_{k k}\left(1-x_{k k}\right)}{\sum_{\ell \neq k} G_{\ell k} p_{\ell}+1} \geq t & \forall k \in \mathcal{K}, \\
\frac{G_{m k} p_{m}+M_{m k}\left(1-x_{m k}\right)}{\sum_{\ell \neq m} G_{\ell k} p_{\ell}+1} \geq t & \forall k \in \mathcal{K}, \forall m \neq k, \\
\frac{G_{k k} p_{k}+M_{m k}\left(1-x_{m k}\right)}{\sum_{\ell \neq m, k} G_{\ell k} p_{\ell}+1} \geq t & \forall k \in \mathcal{K}, \forall m \neq k, \\
\sum_{m=1}^{K} x_{m k}=1 & \forall k \in \mathcal{K} .
\end{array}
$$

In what follows, we will explain the operational meaning of each condition in (9). The parameters $M_{m k}$ are calculated considering the worst-case scenario, where the power term in the numerator is 0 , whereas all the power terms appearing in the denominator are equal to their upper bound. Then, we have

$$
M_{m k}:=U\left(\sum_{\ell \neq m} G_{\ell k} P_{\ell}+1\right)
$$

where $U$ is the upper bound on the SINR level determined in (7).

The $k$ th inequality in (9a) defines the SINR constraint, when the $k$ receiver is not performing IC. This event corresponds to the conventional scenario discussed in Section 2, where all interference is treated as noise. Focusing on the $k$ th link, this is true when $x_{k k}=$ 1. Then, the respective inequality in (9a) is the same as (4). In that respect, problem (8)-(9) is a generalization of (3)-(4) since it includes the latter as a special case.

Each pair of inequalities (9b)-(9c) corresponds to a possible event of single-stage IC. Specifically, the direct link of interest is defined by the index $k$, whereas the interference term that is subtracted from the received signal is denoted by the index $m \neq k$. The $k$ th receiver is able to subtract the interference term of the $m$ th transmitter only when this signal is decodable. The prerequisite SINR constraint is given in (9b), where the desired signal is accounted as interference. It is a necessary condition to enable cancellation of the interference term of the $m$ th transmitter from the sum, so that the constraint for decoding the desired signal can now be defined as in $(9 \mathrm{c})$. Note that the denominator in $(9 \mathrm{c})$ contains a term less than the respective of (9a); the interfering signal from the $m$ th transmitter.

Equation (9d) ensures that each receiver selects at most one of the possible single-stage IC scenarios. The choice $x_{k k}=1$ corresponds to the event that it is not possible for receiver $k$ to cancel any interference term, whereas $x_{m k}=1$ means that it cancels the $m$ th term first before proceeding in decoding of the desired signal.

Note that the optimization problem (8)-(9) is always feasible. A trivial solution is the one of the conventional (without IC) power control problem (3)-(4) to which it falls back for $\left\{x_{k k}=1\right\}_{k \in \mathcal{K}}$ and $\left\{x_{m k}=0\right\}_{m \neq k, k \in \mathcal{K}}$.

It can be easily verified that (8)-(9) is quasi-linear, similarly to (3)-(4). Hence, the bisection algorithm that was sketched in the previous section can be used to determine the maximum feasible SINR level. Since the denominator of the fraction in the left-hand-side term of (9b) is positive, we can equivalently rewrite the constraint, for a given value of $t:=T$, as

$$
\sum_{\ell=1}^{K} A_{\ell k} p_{\ell}+M_{m k} x_{m k} \leq B_{m k}
$$

where $B_{m k} \triangleq M_{m k}-T$ and

$$
A_{\ell k} \triangleq \begin{cases}-G_{m k} & \text { if } \ell=m \\ T G_{\ell k} & \text { if } \ell \neq m\end{cases}
$$

Clearly, inequality (11) is linear to all (continuous and binary) variables. It is easily seen that setting $m=k$ in (11), we get the respective linear representation of (9a). For compactness, we jointly represent (9a) and (9b) with (11) $\forall m \in \mathcal{K}$.

By the same token, we equivalently rewrite (9c) as

$$
\sum_{\ell=1}^{K} C_{\ell k} p_{\ell}+M_{m k} x_{m k} \leq B_{m k}
$$

where

$$
C_{\ell k} \triangleq \begin{cases}0 & \text { if } \ell=m, \\ -G_{k k} & \text { if } \ell=k, \\ T G_{\ell k} & \text { if } \ell \neq m, k .\end{cases}
$$

The feasibility problem that is solved in every iteration of the bisection algorithm, is formulated by replacing $(9 a)-(9 b)$ and $(9 c)$ with (11) and (13) respectively

$$
\begin{array}{ll}
p_{k} \in\left[0, P_{k}\right] & \forall k \in \mathcal{K}, \\
x_{m k} \in\{0,1\} & \forall k \in \mathcal{K}, \forall m \in \mathcal{K}, \\
\sum_{\ell=1}^{K} A_{\ell k} p_{\ell}+M_{m k} x_{m k} \leq B_{m k} & \forall k \in \mathcal{K}, \forall m \in \mathcal{K}, \\
\sum_{\ell=1}^{K} C_{\ell k} p_{\ell}+M_{m k} x_{m k} \leq B_{m k} & \forall k \in \mathcal{K}, \forall m \neq k, \\
\sum_{m=1}^{K} x_{m k}=1 & \forall k \in \mathcal{K} .
\end{array}
$$

The conditions in (15) denote a feasibility problem in standard MILP form. Regarding computational complexity, there is a fundamental difference with the conventional case. Since some of the variables are binary, the problem is combinatorial. However, due to the linearity of the problem, we can find the global-optimal solution efficiently, for the vast majority of instances, using standard algorithms.

\section{COMPUTATIONAL COMPLEXITY}

In this section, we show that the max-min power control problem with single-stage IC (8)-(9) is NP-hard. Specifically, we claim that the feasibility problem (15) is NP-complete, because it is a decision problem with a yes/no answer. Since the decision problem is NPcomplete, the optimization version of the problem, i.e., (8)-(9), is NP-hard by definition. This is strong result, since without IC the max-min power control problem (5)-(6) is quasi-LP.

Theorem 1. The feasibility problem, defined by the constraints (15) for a given SINR threshold T, is NP-complete.

Proof. Due to space limitation, we restrict the presentation to a sketch of the proof and leave out some of the technical details. The polynomial-time reduction in the proof is based on the well-known 3-satisfiability (3-SAT) problem, which is NP-complete [7]. In 3SAT, we are given a number of boolean variables, each can take value true or false, and a set of clauses, each consisting in three literals, where a literal is either a variable or its negation. A clause is true if any of its literals is true. The task is to determine if there is an assignment of boolean values to the variables, such that all clauses become true.

Given a 3-SAT instance with $N$ variables and $D$ clauses, we define an equivalent instance of the decision problem (15) as follows. For the clauses $1, \ldots, D$, we define two links $1, \ldots, D, D+$ $1, \ldots, 2 D$. Each $d^{\prime} \in\{D+1, \ldots, 2 D\}$ is coupled with link $d=$ $d^{\prime}-D$ only, i.e., the gain values between $d^{\prime}$ and the remaining links (including those defined below) are negligibly small. For links $d^{\prime}$ and $d=d^{\prime}-D$, the gain values $G_{d^{\prime} d^{\prime}}, G_{d^{\prime} d}, G_{d d^{\prime}}$ are set such that: 
1) link $d^{\prime}$ can be active only if its power meets a minimum value (to overcome noise), 2) when $d^{\prime}$ transmits using this power, link $d$ can cancel the interference of $d^{\prime}$ even under worst-case interference from the other links, and 3) if link $d$ does not cancel the transmission of $d^{\prime}$, then $d$ can not be active if $d^{\prime}$ is active.

Next, we add link set $\{1, \ldots, N\}$ representing the boolean variables and their negations. For each link $n$ and the link $\hat{n}$ representing the negation, the gain values are set such that both can be admitted only if exactly one of them cancels the other. In addition, the transmission being canceled must use the maximum power, and the other transmission must use the minimum power (derived from the noise effect). Each link $d$ representing a clause receives interference only from the links corresponding to the three literals of the clause. By construction, link $d$ will not cancel any of these interfering transmissions. Moreover, the gain values between the three interfering transmissions and $d$ are set such that link $d$ can be active only if at least one of the three transmissions uses minimum power. Consequently, the feasibility problem (15) that we define has a yes-answer if and only if this is true for the 3-SAT instance. Hence the conclusion.

\section{SIMULATION EXAMPLE}

We present a simulation result to illustrate the potential of IC. We consider a small network of $K=4$ links. The transmitters are placed at the corners of a square with area $100^{2}$. The receivers are uniformly placed within square "cells" of area $100^{2}$. The normalized channel gains are determined by the geometric propagation loss model, i.e., $G_{\ell k}=d_{\ell k}^{-\alpha} / \sigma^{2}$, where $d_{\ell k}$ is the link distance among the $\ell$ th transmitter and the $k$ th receiver, $\alpha=4$ is the attenuation factor, and $\sigma^{2}=10^{-10}$ is the noise variance. We chose this scenario because it yields direct channels with larger gains than the crosstalk channels. Hence, decoding an interfering signal before the desired signal is nontrivial. The power bounds are set to $\left\{P_{k}=10\right\}_{k \in \mathcal{K}}$. We use the GNU linear programming kit (GLPK) package to solve the MILP feasibility problem (15).

In Fig. 1, the upper plot depicts the maximum achievable common SINR, for 50 random instances ${ }^{2}$ of the network topology. Both the solution of the novel (8)-(9) and conventional (5)-(6) problem are shown. It is seen that in around half of the instances, IC is possible and boosts the performance by several $\mathrm{dB}$. The lower plot shows the average transmit power per link. When IC is possible, the transmit power is in most cases increased to enable higher SINR.

\section{CONCLUDING REMARKS}

We proposed an optimization framework that jointly determines whether IC is possible and controls the transmit powers accordingly. We evidenced significant gains even with single-stage IC. In practice, IC is not a trivial problem mostly owing to the synchronization issues involved. Specifically, the receiver must estimate, with sufficient accuracy, the channels between itself and all transmitters whose signals it is trying to decode. Moreover, unless the transmitters are perfectly synchronized in time and frequency there will be residual frequency and timing offsets that would have to be estimated at the receivers as well, before the decoding can take place. Moving receivers also complicate the picture owing to Doppler effects that introduce frequency offsets at the receiver, even in the event that the transmitters would be perfectly synchronized. Here, we assumed that IC is possible without significant performance impairments resulting from offsets. Hence, the results provided actually constitute upper bounds on what would be achievable in practice.

${ }^{2}$ Average results are included in the journal version under preparation.
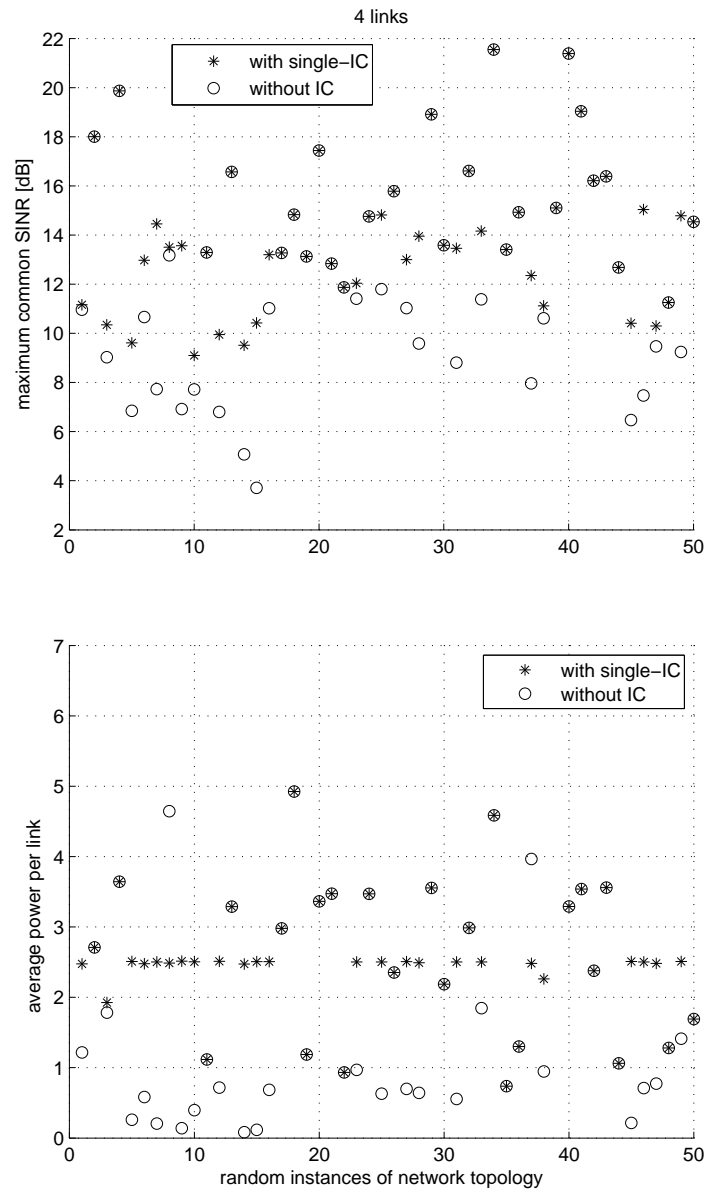

Fig. 1. Max-min power control with and without IC

\section{REFERENCES}

[1] X. Shang, G. Kramer, and B. Chen, "A new outer bound and the noisy-interference sum-rate capacity for Gaussian interference channels," IEEE Trans. Inf. Theory, vol. 55, no. 2, pp. 689-699, Feb. 2009.

[2] V. S. Annapureddy and V. V. Veeravalli, "Gaussian interference networks: sum capacity in the low interference regime and new outer bounds on the capacity region," IEEE Trans. Inf. Theory, vol. 55, no. 6, p. 3032-3050, Jun. 2009.

[3] T. Cover and J. Thomas, Elements of Information Theory. New York: Wiley, 1991.

[4] S. Stańczak, M. Kaliszan, N. Bambos, and M. Wiczanowski, "A characterization of max-min SIR-balanced power allocation with applications," in Proc. IEEE ISIT, 2009, pp. 2747-2751.

[5] P. Björklund, P. Värbrand, and Di Yuan, "Resource optimization of spatial TDMA in ad hoc radio networks: A column generation approach," in Proc. IEEE INFOCOM, 2003, pp. 818-824.

[6] E. Karipidis, E. G. Larsson, and K. Holmberg, "Optimal scheduling and QoS power control for cognitive underlay networks," in Proc. IEEE CAMSAP, 2009, pp. 408-411.

[7] M. R. Garey and D. S. Johnson. Computers and Intractability: A Guide to the Theory of NP-Completeness. W. H. Freeman, 1979. 